\title{
Zusammenarbeit innerhalb der FMH Services zwischen den Partnern der FMH Treuhand und der FMH Insurance Services
}

Urs Arpagaus, Adrian Hartmann

Korrespondenzen: Urs Arpagaus, dipl. Betriebsökonom FH FMH Insurance Services Steinackerstrasse 7 CH-9214 Kradolf

Adrian Hartmann, dipl. Treuhandexperte FMH Services Treuhand Marktplatz 6 CH-8570 Weinfelden
Ziel der FMH Services ist es, ihren Mitgliedern eine möglichst ganzheitliche Beratung/Struktur anzubieten. In den Bereichen Treuhand, Buchführung, Steuerplanung, Finanzierung, Finanzberatung und Versicherung gilt dies ganz besonders für die Partner FMH Treuhand und FMH Insurance Services. Wie kann nun eine $\mathrm{Zu}$ sammenarbeit zwischen diesen beiden Beratern aussehen und was darf der Kunde erwarten?

\section{Fachlicher Aspekt und Aufgaben der Berater}

Treuhänder und Finanzberater müssen zusammen ein «Basisnetzwerk» bilden. Aufgrund der ineinander übergreifenden Sachgebiete bzw. Beratungsfelder ergeben sich zwangsläufig Überschneidungen. Als Beispiele seien hier erwähnt: Vorsorge $\leftrightarrow$ Steuern, Investitionen $\leftrightarrow$ Finanzen, Liquidität $\leftrightarrow$ Finanzanlagen. Zudem ist es so, dass sich der eine Berater, zumindest in groben Zügen, auch im Fachbereich des anderen Beraters auskennt. Somit kann jede der beiden Parteien durchaus auch Aufgaben der anderen Partei übernehmen bzw. beurteilen. Dies führt im Basisnetzwerk automatisch zum 4-Augen-Prinzip.

Gemeinsam müssen die beiden Berater folgende Aufgabenblöcke erfüllen:

\section{Strategisch-planerische Arbeiten}

- Persönliche Lebensplanung, Besonderheiten, Meilensteine des Kunden;

- betriebswirtschaftliche Planung;

- Liquiditätsplanung;

- Planung der Vorsorge-, der Versicherungslösungen usw.

\section{Implementorische Arbeiten}

- Steuerliche Optimierungsmöglichkeiten;

- Bilanzpolitik (Bilanzwahrheit und -klarheit);

- Kennzahlenvergleiche und -interpretation;

- Performancevergleich (für Versicherungs-, Finanz- und Immobilienanlagen);

- Sicherstellung Liquidität für Einkäufe, Planwechsel bei Pensionskassen, Investitionen usw.

\section{Kurzfristig-taktische Arbeiten}

- Vermögensumschichtungen, Auflösung Säule 3a, Finanztransaktionen;

- Verhandlungen mit Banken, Behörden und Versicherungen in Krisensituationen;

- Schadenfälle jeglicher Art.

Aus jedem dieser drei Aufgabenblöcke heraus entstehen konkrete Massnahmen. Damit der Kunde keine negativen Überraschungen erlebt, müssen diese Aufgaben in gemeinsamer $\mathrm{Zu}$ sammenarbeit abgeklärt und bearbeitet werden. Die getroffenen Entscheidungen und Massnahmen müssen zwingend abgestimmt sein auf die individuelle Situation wie z. B. die Risikoneigung jedes einzelnen Kunden.

Eine Maxime berühmter Feldherren lautet: «Handle stets so, dass Du Optionen behältst.» Die Qualität der Massnahmen in den jeweiligen Bereichen ist nur so gut, wie sie es dem Kunden auch in Zukunft erlauben, sich auf veränderte Bedingungen einzustellen bzw. schnell und kontrolliert auf neue Situationen zu reagieren.

\section{Zeitlicher Aspekt}

Aus den unterschiedlichen $\mathrm{zu}$ bearbeitenden Tätigkeitsfeldern ergibt sich zwangsläufig auch ein unterschiedlicher Beratungszyklus. Der Treuhänder wird z.B. mindestens einmal jährlich zu Besuch weilen, da die Buchhaltung bzw. die Steuererklärung jährlich zu erstellen sind. Somit wird dieser Beratungsrhythmus von aussen vorgegeben.

Der Finanz- und Versicherungsberater wird bei Mandatsübernahme sicher innert kurzer Zeit mehrmals ein Beratungsgespräch führen. Anschliessend, wenn Versicherungen und Finanzanlagen strukturiert und angepasst sind, fehlt jedoch in aller Regel ein von aussen diktierter Rhythmus.

In welchen Zeitabschnitten der Kunde betreut werden muss, hängt aber von vielen Faktoren ab. Zwei wichtige Aspekte sind die Komplexität des Mandates und die Situation, in der sich der Kunde gerade befindet (aktueller Lebensab- 
schnitt, Notsituation usw.). Ab einer gewissen Grösse bzw. Komplexität des Mandates ist jedoch sicher ein gemeinsamer Check-up ein- bis zweimal jährlich notwendig.

Aus den im vorstehenden Abschnitt erwähnten Aufgabenblöcken heraus entstehen, wie erwähnt, konkrete Massnahmen. Diese Massnahmen werden in einer «To-do-Liste» zusammengefasst und zeitlich geordnet. Zudem ist eine Unterteilung in «Muss» und «Kann» wünschenswert. Diese To-do-Liste muss stetig nachgeführt und auf ihre Relevanz hin überprüft werden und gibt somit den Beratungsrhythmus bei grösseren oder komplexeren Mandaten vor.

\section{Formen der Zusammenarbeit}

Der Kunde sollte vorab den jeweiligen Berater über die Zusammenarbeit mit dem anderen Berater informieren. Folglich muss er die beiden Partner von ihrer Verschwiegenheitspflicht einander gegenüber entbinden. Mit dieser Voraussetzung können die beiden Partner nun die notwendigen Informationen austauschen. Dieser Informationsaustausch kann in regelmässigen Treffen zwischen den Beratern stattfinden. Oder der Treuhänder informiert jeweils kurz den Finanz- und Versicherungsberater, wenn der Jahresabschluss fertiggestellt ist. Umgekehrt informiert der Finanz- und Versicherungsberater den Treuhänder, falls Änderungen im Versicherungsportefeuille und in den Finanzanlagen anstehen.

Die Führung eines solchen Teams obliegt jedoch Ihnen als Kunde. Fordern Sie Ihr Team, indem Sie z. B. das koordinierte Vorgehen mit den Partnern besprechen, den Ablauf kontrollieren und sich das Resultat bzw. den Erfolg präsentieren lassen.

Wie Sie das gestalten, hängt im wesentlichen auch von Ihrem persönlichen Naturel ab und natürlich vom Vertrauen, das Sie gegenüber Ihrem Team gebildet haben.

Ein wichtiger Aspekt für die erfolgreiche $\mathrm{Zu}$ sammenarbeit dieser beiden Partner ist jedoch ein Verständnis für die Antriebskräfte, denen ein Treuhänder und ein Finanzberater ausgesetzt sind. Dies beginnt bei der Gleichbehandlung der
Partner und endet bei der finanziellen Entschädigung der Dienstleistungen.

\section{Ziele der Zusammenarbeit}

Das Ziel beider Partner ist eine optimale, kundenorientierte Beratung. Diese soll individuell und auf die Bedürfnisse zugeschnitten sein. Hieraus ergibt sich eine kontinuierliche, langfristige Zusammenarbeit bzw. Beratertätigkeit mit einem mittel- und langfristigen Beratungshorizont.

\section{Vorteile für den Kunden}

Nicht nur ein, sondern zwei Ansprechpartner ermöglichen erst eine umfassende und gesamtheitliche Beratung. Der Kunde erhält eine höhere Sicherheit, da er jeweils die Meinung des anderen Partners zu einer Problemstellung oder Lösungsmöglichkeit einholen kann. Zudem sehen vier Augen mehr als zwei.

Es liegt auf der Hand, dass nicht alles planbar ist, aber ein eingespieltes Team, das sich mit der Situation des Kunden bestens auskennt, kann unvorhergesehene Ereignisse besser meistern. Schnellschüsse oder uninformierte Entscheidungen (als Resultat einer «Einweg-Produkte-Beratung») kosten nachträglich Geduld, Ärger und häufig auch viel Zeit und Geld.

\section{Fazit}

Die hier aufgestellten Vorgaben sind natürlich äusserst komplex und bedingen ein qualifiziertes und interdisziplinär starkes Team mit Erfahrung, geistiger Flexibilität, absoluter Unabhängigkeit und nicht zuletzt gegenseitigem Respekt. Denn bei Bedarf müssen ja auch noch andere, zusätzliche Partner in dieses Basisnetzwerk miteingeschlossen werden können, wie z.B. ein Rechtsanwalt, ein Immobilienfachmann, Produktspezialisten von Finanzinstituten oder ein Architekt.

Unabhängigkeit, Qualität und Kontinuität sind die tragenden Pfeiler einer Zusammenarbeit oder philosophisch von etwas anderer Seite betrachtet: «Wo das Bewusstsein schwindet, dass jeder Mensch uns als Mensch etwas angeht, kommen Kultur und Ethik ins Wanken.» 